\title{
DRUG RELEASE OF NANO-HYDROXYAPATITE BIOLOGICAL SUBSTITUTE LOADED WITH ISONIAZID AND RIFAMPIN IN VITRO AND ANIMAL MODELS OF SPINAL TUBERCULOSIS
}

\author{
ZE-CHENG CAI ${ }^{1, \#, ~ J U A N ~ L I U ~}{ }^{2, \#}$, HE MA ${ }^{3}$, RONG MA ${ }^{4}$, ZHEN CHEN ${ }^{4}$, \\ JIAN-QUN ZHANG ${ }^{4}$, XIAO-YIN LIU ${ }^{4}$, and ZHAO-HUI GE** \\ ${ }^{1}$ Ningxia Medical University, Yinchuan, Ningxia, 750004, China \\ ${ }^{2}$ Department of Thoracic Surgery, General Hospital of Ningxia Medical University, \\ Yinchuan, Ningxia, 750000, China \\ ${ }^{3}$ Shenzhen Baoan People's Hospital, Shenzhen, 518101, China \\ ${ }^{4}$ Department of Orthopedics, General Hospital of Ningxia Medical University, 750000, China
}

\begin{abstract}
Spinal tuberculosis drug efficacy studies have shown that a drug's clinical efficacy mainly depends on its concentration in the target tissue. To improve the local drug concentration of spinal tuberculosis, we studied the release of isoniazid and rifampin-loaded nano-hydroxyapatite biological substitutes in vitro and in animal models. In vitro experiments, isoniazid produced burst release, releasing $44.57 \%$ and $98.31 \%$ of drug at $24 \mathrm{~h}$ and four weeks, respectively. The rifampin release was relatively stable without obvious burst release. The release time of rifampin was longer, with $98.26 \%$ release in the $12^{\text {th }}$ week and the remaining $1.74 \%$ in biological substitute. In animal models, isoniazid concentration in biological substitutes of the vertebral body and paravertebral muscle gradually decreases and can be maintained until the $8^{\text {th }}$ week. The drug concentration in inferior vena cava reached its maximum at $72 \mathrm{~h}$ and then gradually decreased. No drug concentration was detected in the second week. The rifampin concentration in the vertebral body and parallel muscle reached the maximum in the first week, then decreased gradually and maintained to the $12^{\text {th }}$ and $8^{\text {th }}$ weeks. No drug concentration was detected in inferior vena cava after $72 \mathrm{~h}$. Nano-hydroxyapatite biological substitute loaded with isoniazid and rifampin can be degraded stably in vitro and has good sustained-release properties. In animal models, antituberculosis drugs can be released continuously in the long term.
\end{abstract}

Keywords: spinal tuberculosis, isoniazid, rifampin, biological substitute, sustained-release

Spinal tuberculosis drug efficacy research has demonstrated that a drug's clinical efficacy primarily relies on its target tissue concentration. Therefore, whether antituberculosis drugs' distribution in spinal tuberculosis can be effectively distributed is the key towards spinal tuberculosis treatment (1). Studies have revealed that due to sequestrum and sclerotic bone formation at spinal tuberculosis focus, conventional antituberculosis drugs such as isoniazid (INH) and rifampin (RFP) are challenging to reach effective concentrations at the focus. Several studies have recently tried to treat spinal tuberculosis by local drug delivery after constructing local sustainedrelease materials, but owing to various materials, the effects are quite different (2-3).

As the main inorganic minerals for filling bone defects, hydroxyapatite and calcium sulfate have been extensively used in studying bone substitutes
(4-5). Based on previous studies on the biomechanics of biological substitutes (6), we further explored its drug release performance and degradation in vitro and verified its drug release characteristics in animal models. This was to provide some theoretical basis for applying biological substitutes in the local treatment of spinal tuberculosis.

\section{MATERIALS AND METHODS}

This study has been approved by the research ethics review committee of the General Hospital of Ningxia Medical University (No.2019-035).

\section{Chemicals and reagents}

Nano-hydroxyapatite (nHA) was prepared in the laboratory, and calcium sulfate (CS) was purchased from Sigma-Aldrich (St. Louis, MO, USA).

\#These authors contributed equally to this work,

*Corresponding author: e-mail: myovid@126.com 
Chitosan (CTS), calcium nitrate tetrahydrate (analytical reagent, $\left.\mathrm{Ca}\left(\mathrm{NO}_{3}\right)_{2} \cdot 4 \mathrm{H}_{2} \mathrm{O}\right)$, polyacrylic acid (analytical reagent, PAA), and ammonium phosphate (analytical reagent, $\left(\mathrm{NH}_{4}\right)_{2} \mathrm{HPO}_{4}$ ) were obtained from SINOPHARM (Beijing, China). INH and RFP were purchased from NIFDC (Beijing, China) with batch numbers of 100578-201502 and 110757-201502, respectively. Sodium dihydrogen phosphate and acetic acid (analytical reagent, $\mathrm{CH}_{3} \mathrm{COOH}$ ) were provided by Nanjing Chemical Reagent Ltd (Nanjing, China). Methanol and acetonitrile (HPLC GRADE) were supplied by Fisher Scientific (Massachusetts, USA). The following instruments were utilized: high-speed refrigerated centrifuge (Centrifuge 5810R, Eppendorf, Germany), high-performance liquid chromatography (HPLC) (1200 series, Agilent, USA), scanning electron microscope (SEM) (S-3400N, HITACHI, Japan), X-ray diffractometer (XRD-6000, Shimadzu, Japan), and Fourier transform infrared spectrometer (TENSOR37, Bruker, Germany). New Zealand Rabbits were provided by Shaanxi Xingping Tianrui experimental animal breeding factory, China.

\section{Preparation of $\mathbf{n H A}$}

nHA was prepared by the chemical precipitation method. According to the ratio of $n(\mathrm{Ca}) / \mathrm{n}(\mathrm{P})=1.67$, calcium nitrate tetrahydrate $(5.93 \mathrm{~g})$ and ammonium phosphate $(2.00 \mathrm{~g})$ were completely dissolved in distilled water forming solution A and solution B, respectively. Under vigorous stirring (1000 r/min), solution B was slowly dropped into solution A, and a small amount of PAA solution was then added, followed by adjusting reaction solution $\mathrm{pH}$ at about 10 with $25 \%$ ammonia. After dropping, the reaction system temperature was kept, and stirring lasted for three hours. After standing at room temperature for $12 \mathrm{~h}$, the reactants were filtered and washed with a suction filter and continuously washed with distilled water until $\mathrm{pH} \approx 7$. The powder was then dried into a vacuum oven.

\section{Fabrication of biological substitute}

According to the preparation method in the early stage of the research group (6), the weight ratio between nHA : CSH : INH : RFP was set as $2.0: 8.0: 0.2: 0.2$. All operations were performed under dark conditions. nHA (0.20 g), CSH (0.80 g), INH (0.02 g), and RFP (0.02 g) were charged into a $5 \mathrm{~mL}$ centrifuge tube, mixed thoroughly with a vortex shaker, and used as the solid phase of a biological substitute. CTS (2.00 g) was dissolved in $100 \mathrm{~mL}$ of $1 \%$ acetic acid solution as liquid phase. According to the solid phase ratio to the liquid phase of 0.9 , the prepared phases were fully mixed and then injected into a hollow cylindrical mold with $12 \mathrm{~mm}$ height and $6 \mathrm{~mm}$ diameter. After $3 \mathrm{~min}$ of setting, it was taken out from the mold, dried, and cured for more than 3 days to obtain a biological substitute loaded with INH and RFP. After that, the prepared biological substitute was stored in a refrigerator at $4^{\circ} \mathrm{C}$ in dark.

\section{In vitro release of biological substitute}

All operations were conducted under dark settings. First, five biological substitute samples were prepared, and each sample was soaked in $10 \mathrm{~mL}$ PBS. After that, the temperature was controlled at $37^{\circ} \mathrm{C}$ by a water bath, and the experiment's time points were set to $24 \mathrm{~h}, 72 \mathrm{~h}$ and 1, 2, 4, 6, 8, 10, $12 \mathrm{w}$. At various time points, the five biological substitutes were taken out and placed in new $10 \mathrm{~mL}$ PBS to continue soaking. These steps were repeated. After removing the biological substitute, the leaching solution was vortexed on a vortex shaker for $3 \mathrm{~min}$ and filtered with a $0.22 \mu \mathrm{m}$ microporous filter, and concentration of INH and RFP was detected by HPLC. At the final time point, the biological substitute was transferred into a homogenizer, and $10 \mathrm{~mL}$ methanol aqueous solution (methanol : water $=1: 1$ ) was added for homogenization. The purpose is to detect the drug concentration that has not been soaked in the biological substitute. After completing the above operations, the cumulative drug release amount and cumulative drug release percentage were calculated to observe the sustained drug release.

\section{Establishment of spinal tuberculosis model rabbit}

One month before establishing the animal model, according to previous methods (7), each experimental rabbit requires to be injected with $0.1 \mathrm{~mL}$ of Freund's complete advance (subcutaneous tissue on the back of the neck). One month later, the injection site appeared yellowish induration with a diameter of about $0.8-1.0 \mathrm{~cm}$, without ulceration and bleeding, which is an effective sensitization. Sensitization aims to induce antibodies' production against mycobacterium tuberculosis in animals and reduces blood dissemination after planting the $\mathrm{H}_{37} \mathrm{R}_{\mathrm{v}}$ standard strain. The experimental rabbits with successful sensitization underwent surgery to establish a spinal tuberculosis model. No food and drink were supplied before surgery, and skin preparation was done on the surgical area. A 3\% sodium pentobarbital solution was used to inject anesthesia through the ear vein, and successful anesthesia showed corneal response disappearance, slow and steady breathing rate, and muscle tone decrease. Each rabbit was fixed in the right decubitus position, and the disinfection was completed. Through the abdominal approach on transverse 
process side of vertebral body, L4-5 vertebral body was exposed. At the upper part of L5 vertebral body, $0.5 \mathrm{~cm}$ away from L4/L5 intervertebral disc, a bone hole about $5 \mathrm{~mm}$ deep and $3 \mathrm{~mm}$ in diameter was made with a bone drill along the left posterior to right anterior direction. After sufficient hemostasis, a gelatin sponge was filled. A $0.1 \mathrm{~mL}$ of mycobacterium tuberculosis (MTB) suspension (human MTB, $\mathrm{H}_{37} \mathrm{R}_{\mathrm{V}}$ standard strain) was slowly injected with a $1 \times 107 \mathrm{CFU} / \mathrm{mL}$ concentration on the gelatin sponge with a syringe and then closed the surgical incision layer by layer. After feeding for two months, the animal model was subjected to radiological examination.

\section{Implant biological substitutes in animal models}

Two months later, the successfully modeled rabbit was placed on the operating table on its side. After anesthesia was completed, surgical disinfection was completed. From the original surgical incision approach, bone and intervertebral discs destroyed by MTB were revealed, and lesion resection was performed. The resection scope includes abscesses, necrotic granulation, and dead bone (sclerotic bone around the lesion, $2 \mathrm{~mm}$ subnormal bone, and $2 \mathrm{~mm}$ paravertebral muscles). Adjacent vertebral bodies were ground into depressions using a grinding drill, forming a bone groove together with the defect. The pre-cut $6 \times 6 \mathrm{~mm}$ biological substitute was implanted into the bone groove, reinforced with bone wax, and the surgical incision is closed layer by layer.

\section{Material and processing of animal models}

For the experimental rabbits implanted with the biological substitute, samples were taken at 9-time points of $24 \mathrm{~h}, 72 \mathrm{~h}, 1$ week, 2, 4, 6, 8, 10, and 12 weeks after the operation. Three rabbits were taken out at each time point, and $4 \mathrm{~mL}$ of inferior vena cava blood was collected and then left to death by air embolism. From the original surgical incision to expose the area where the biological substitute was implanted, the $2 \mathrm{~mm}$ upper and lower vertebral body and the surrounding $2 \mathrm{~mm}$ paravertebral muscle tissue of the biological substitute were taken. After freezing and drying for $24 \mathrm{~h}$ in dark at $-50^{\circ} \mathrm{C}$, samples were stored in a refrigerator at $-80^{\circ} \mathrm{C}$. Vertebral body: frozen and dried bone tissues were ground in a mortar for about $20 \mathrm{~min}$ until obtaining powder. To $100 \mathrm{mg}$ powder, $1000 \mu \mathrm{L}$ methanol was added to homogenizer for homogenization and extraction, and the homogenized liquid was collected into a centrifuge tube, which was placed on a vortex mixer for $2 \mathrm{~min}$, then centrifuged in a low-temperature centrifuge $\left(-4^{\circ} \mathrm{C}, 1400 \mathrm{r} / \mathrm{min}\right)$ for $10 \mathrm{~min}$, and finally, $200 \mu \mathrm{L}$ supernatant was obtained. Paravertebral muscle: after weighing $300 \mathrm{mg}$, $800 \mu \mathrm{L}$ methanol was added to the homogenizer to homogenize and extract, and the homogenate was collected into a centrifuge tube, vortexed for $2 \mathrm{~min}$, centrifuged for $10 \mathrm{~min}$ in a low-temperature centrifuge, and $200 \mu \mathrm{L}$ of supernatant was taken. Venous blood: $200 \mu \mathrm{L}$ of inferior vena cava blood was taken, and $1 \mathrm{~mL}$ of methanol was added to precipitate protein which was then centrifuged, and $200 \mu \mathrm{L}$ of supernatant was taken. All extracted samples were stored in a refrigerator at $-20^{\circ} \mathrm{C}$.

\section{Chromatographic conditions of HPLC}

For INH, the column was $5 \mu \mathrm{m} 4.6 \times 250 \mathrm{~mm}$ ZORBAX SB-C18, and precolumn was $5 \mu \mathrm{m}$ $4.6 \times 12.5 \mathrm{~mm}$ SB-C18. The mobile phase was sodium dihydrogen phosphate buffer $(0.02 \mathrm{~mol} / \mathrm{L}, \mathrm{pH}$ 6.0) : methanol : acetonitrile $=90: 5: 5$; the injection volume was $20 \mu \mathrm{L}$, the flow rate was $0.9 \mathrm{~mL} /$ min, the column temperature was $25^{\circ} \mathrm{C}$, and the wavelength was $264 \mathrm{~nm}$. For RFP, the column was $5 \mu \mathrm{m} 4.6 \times 150 \mathrm{~mm}$ ZORBAX XDB-C18, and precolumn was $5 \mu \mathrm{m} 4.6 \times 12.5 \mathrm{~mm}$ XDB-C18. The mobile phase was sodium dihydrogen phosphate buffer $(0.02 \mathrm{~mol} / \mathrm{L}, \mathrm{pH} 7.2)$ : methanol $=33: 67$; the injection volume $20 \mu \mathrm{L}$; the flow rate was $1.0 \mathrm{~mL} / \mathrm{min}$, the column temperature was $35^{\circ} \mathrm{C}$, and the wavelength was $330 \mathrm{~nm}$.

\section{Statistical analysis}

All data were expressed as mean \pm standard deviation and analyzed by SPSS 17.0 software. Kruskal Wallis $\mathrm{H}$ test was used to analyze the original data at the same time. Simultaneously, drug concentration in the focus vertebral body, paravertebral muscle, and venous blood was compared between the two samples by the Nemenyi method. When $\mathrm{p}<0.05$, according to the level of $\alpha=0.05$, the concentration of two samples simultaneously is considered statistically significant.

\section{RESULTS}

\section{Characteristics of nHA}

The SEM image shows that the prepared nHA is uniformly dispersed without obvious agglomeration. The particles are needle-shaped or rod-shaped with about $150 \mathrm{~nm}$ long and $20 \mathrm{~nm}$ in diameter, and the size is relatively uniform (Figure 1). The XRD pattern manifests obvious characteristic peaks of the prepared $\mathrm{nHA}$, and its diffraction peaks are in line with the peaks of standard card PDF 09-0432 (Figure 2). The infrared spectrum demonstrates that absorption peak near $3435 \mathrm{~cm}^{-1}$ is characteristic for 
$\mathrm{OH}-$ group, and those at $1032 \mathrm{~cm}^{-1}, 604 \mathrm{~cm}^{-1}$, and $566 \mathrm{~cm}^{-1}$ are vibration peaks of $\mathrm{PO}_{4}^{3-}$ group. The stretching vibration peaks of $\mathrm{CH}_{3}$ and $\mathrm{CH}_{2}$ respectively appear at 2361 and $2334 \mathrm{~cm}^{-1}$ due to PAA addition (to reduce nHA agglomeration effect) during the preparation process. The absorption peak at $1646 \mathrm{~cm}^{-1}$ may be ascribed to $\mathrm{H}_{2} \mathrm{O}$ adsorbed on nHA surface. The absorption peak near $1422 \mathrm{~cm}^{-1}$ may be attributed to $\mathrm{CO}_{2}$ in the surrounding air. All the characteristic peaks are consistent with hydroxyapatite, indicating that nHA with higher purity was prepared (Figure 3).

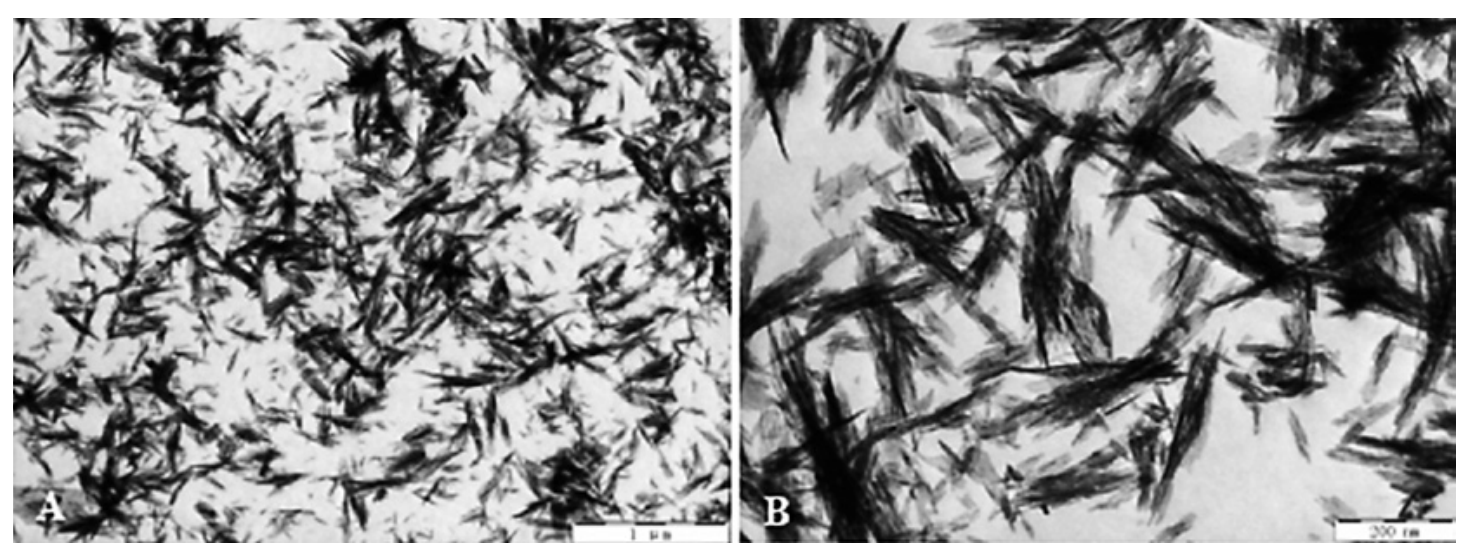

Figure 1. Morphology of nHA under different magnification of SEM. (A) The nHA particles present a uniformly dispersed morphology without obvious agglomeration phenomenon $(\times 5000)$, (B) the particles are needle-shaped or rod-shaped, with uniform size, about $150 \mathrm{~nm}$ in length and $20 \mathrm{~nm}$ in diameter $(\times 10000)$.

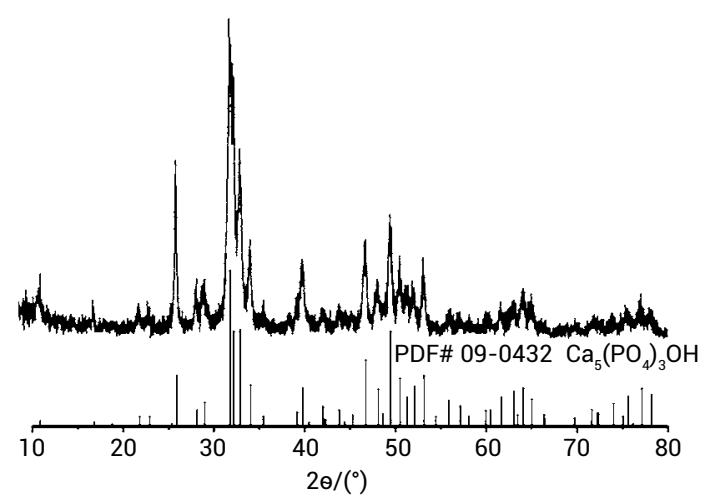

Figure 2. The XRD pattern shows that the characteristic peaks of the prepared nHA are obvious, and its diffraction peaks are in line with the peaks of the standard card PDF 09-0432.

\section{Appearance and SEM image of biological substitute}

The red cylinder was a biological substitute loaded with INH and RFP, and the white cylinder was not loaded with the drug. The volume of each cylinder was $12 \mathrm{~mm}$ high and $6 \mathrm{~mm}$ in diameter (Figure 4). The SEM picture shows that calcium sulfate crystals in the substituted section are short columnar, arranged disorderly, with many interconnected pores, and the crystal surface is unsmooth. nHA and drug are uniformly distributed on calcium sulfate crystals' surface, and the pores are formed in granular or irregular shapes (Figure 5).

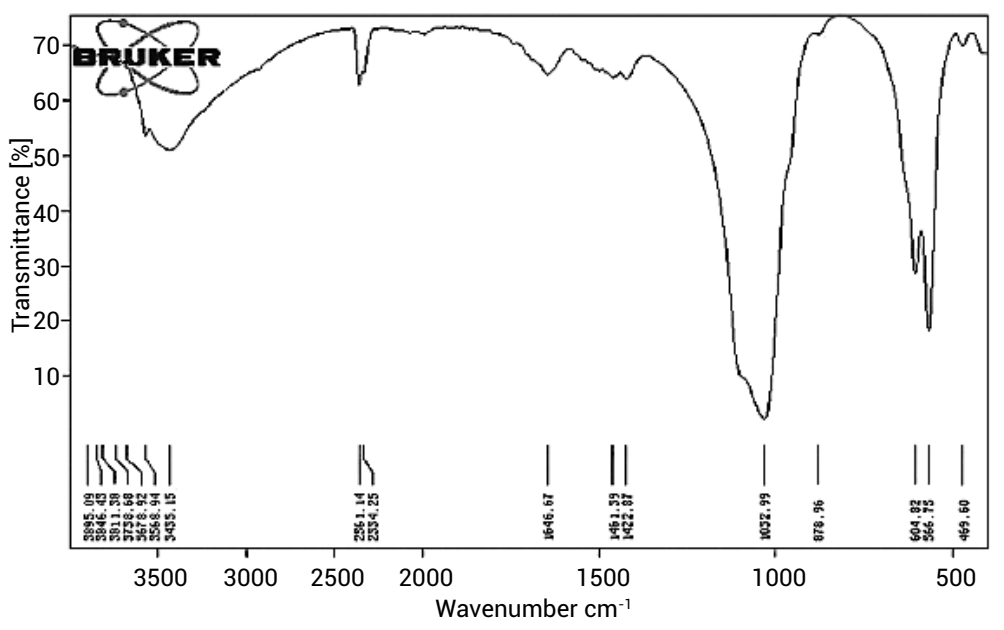

Figure 3. The infrared spectrum shows that all the characteristic peaks are consistent with that of hydroxyapatite. 


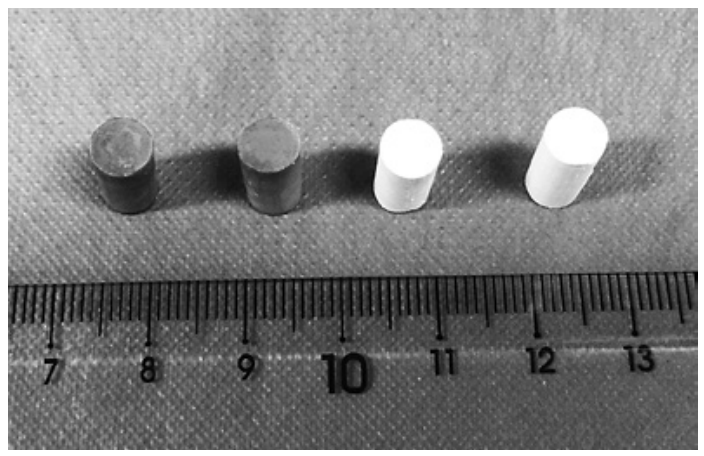

Figure 4. The red cylinder was a biological substitute loaded with INH and RFP, and the white cylinder was not loaded with the drug. The volume of each cylinder was $12 \mathrm{~mm}$ high and $6 \mathrm{~mm}$ in diameter.

Figure 5. The morphology of the cross-section of the biological substitute under different magnifications of SEM. (A) The calcium sulfate crystals are short columnar, arranged disorderly, forming a large number of interconnected pores, and the crystal surface is no longer smooth, (B) nHA and anti-tuberculosis drugs are uniformly distributed on the surface of the crystal, and the pores formed in a granular or irregular shape.
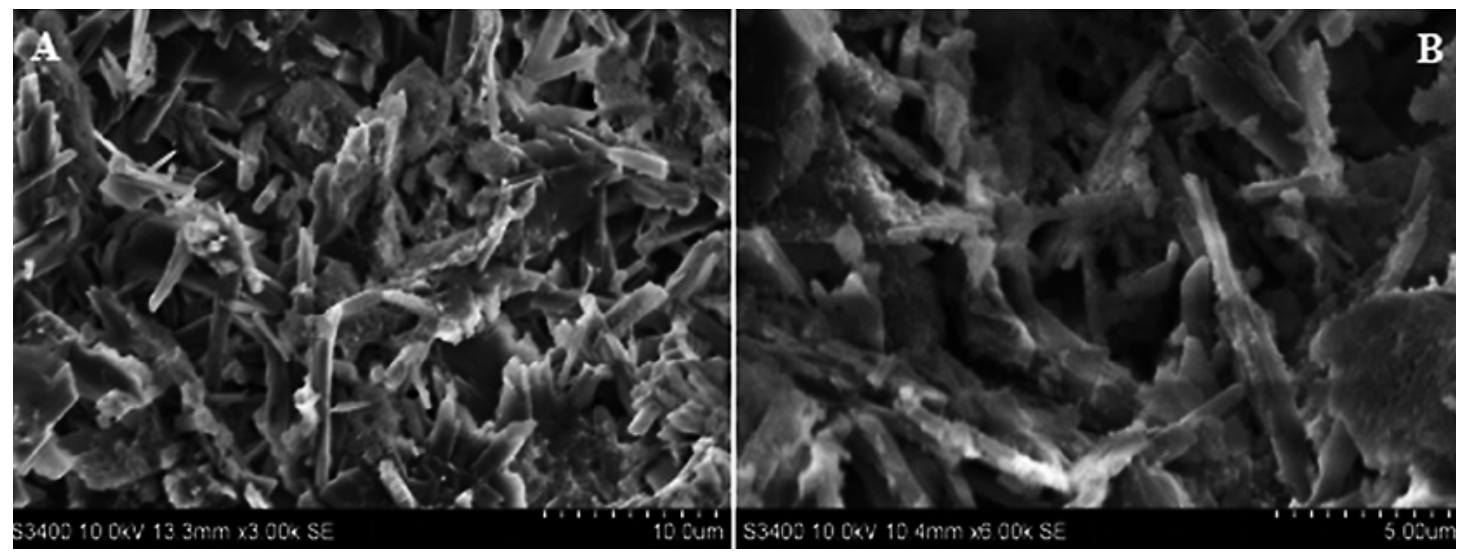

\section{Characteristics of biological substitute in vitro experiments}

With soaking time extension, the biological substitute gradually degrades and decreases in volume. Due to the slow release of RFP (showing red), the red gradually fades until it becomes white (Figure 6). INH produced burst release,

releasing $44.57 \%$ and $98.31 \%$ of the drug at $24 \mathrm{~h}$ and four weeks, respectively. The release time of RFP was longer, releasing $98.26 \%$ in the $12^{\text {th }}$ week, and $1.74 \%$ of RFP remained in biological substitute (Table 1). The release of RFP was relatively stable, and there was no obvious burst release (Figure 7).
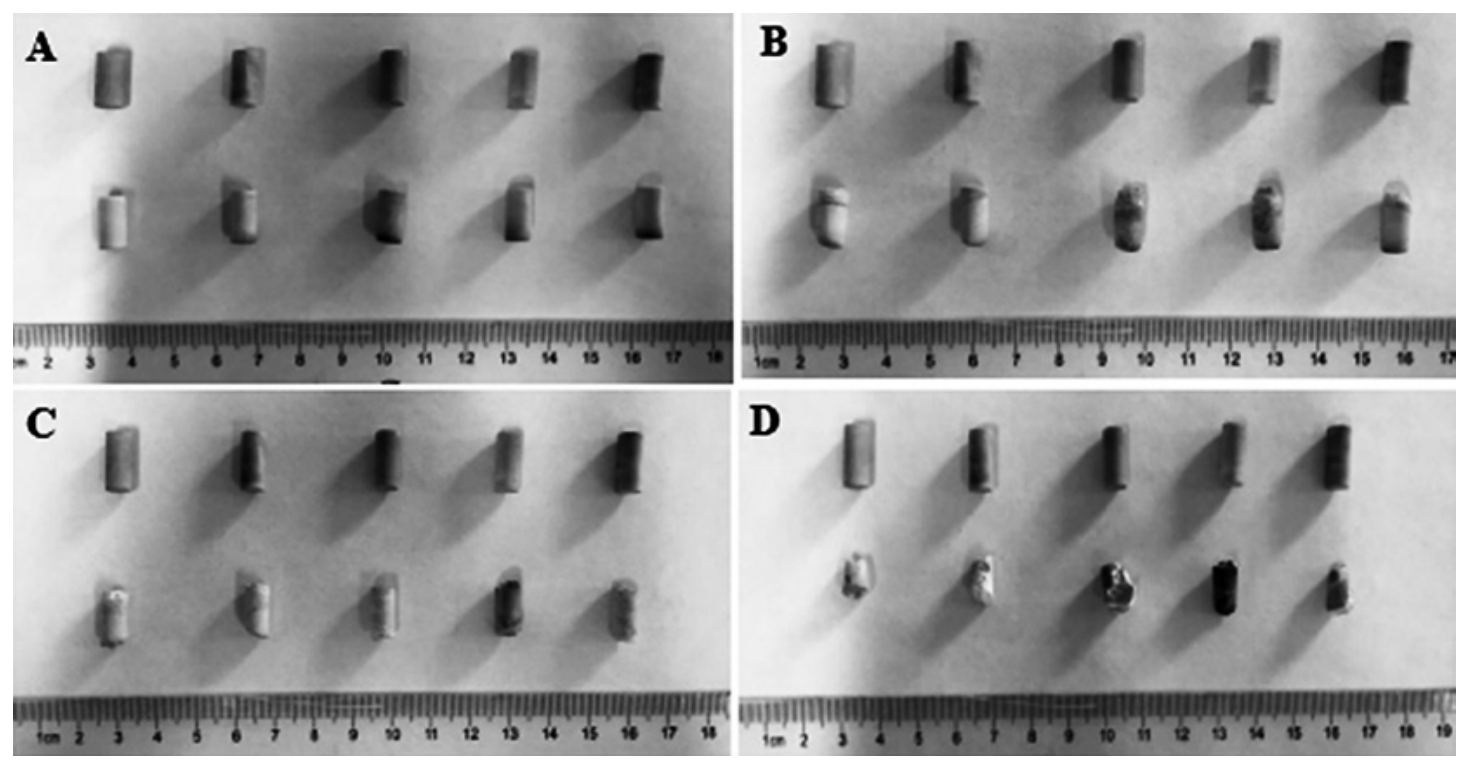

Figure 6. With the extension of the soaking time, biological substitutes gradually degrade, decrease in volume, and gradually fade from red. (A) $72 \mathrm{~h}$, (B) $4 \mathrm{w}$, (C) $8 \mathrm{w}$, (D) $12 \mathrm{w}$. 
Table 1. Drug release concentration and residual drug concentration in biological substitute.

\begin{tabular}{|c|c|c|}
\hline & INH $(\mu \mathrm{g} / \mathrm{mL})$ & RFP $(\mu \mathrm{g} / \mathrm{mL})$ \\
\hline $24 \mathrm{~h}$ & $164.21 \pm 7.97$ & $5.16 \pm 0.73$ \\
\hline $72 \mathrm{~h}$ & $97.80 \pm 6.35$ & $29.48 \pm 1.13$ \\
\hline $1 \mathrm{w}$ & $71.78 \pm 4.35$ & $49.15 \pm 2.40$ \\
\hline $2 \mathrm{w}$ & $20.19 \pm 3.60$ & $45.25 \pm 4.14$ \\
\hline $4 \mathrm{w}$ & $8.23 \pm 0.41$ & $47.97 \pm 2.87$ \\
\hline $6 \mathrm{w}$ & $5.07 \pm 0.82$ & $26.06 \pm 3.25$ \\
\hline $8 \mathrm{w}$ & $1.14 \pm 0.49$ & $2.46 \pm 1.01$ \\
\hline $10 \mathrm{w}$ & - & $0.74 \pm 0.50$ \\
\hline $12 \mathrm{w}$ & - & $0.46 \pm 0.37$ \\
\hline Residual & - & $3.67 \pm 0.78$ \\
\hline
\end{tabular}

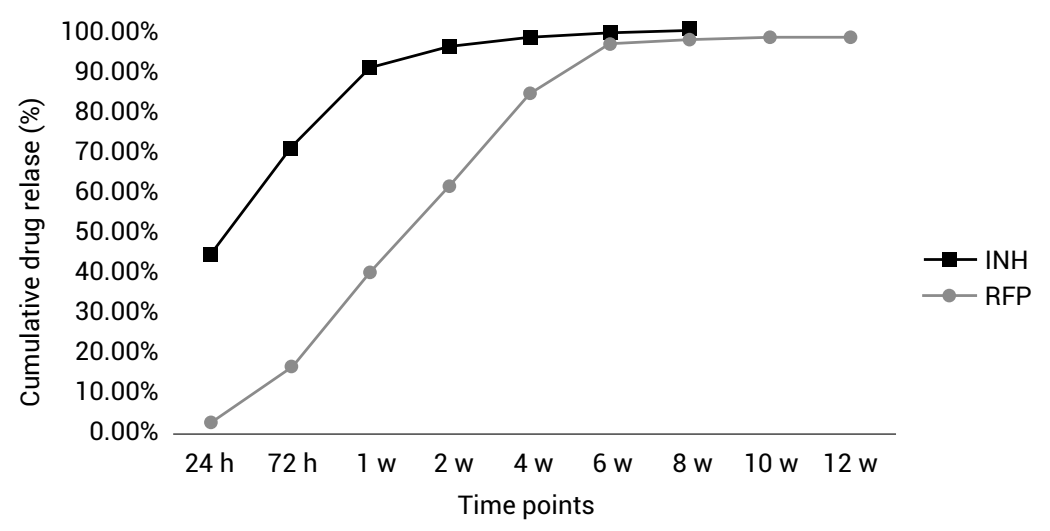

Figure 7. Cumulative drug release curve in biological substitute. The release of RFP is relatively stable compared to INH.

\section{Postoperative observation}

All animals were anesthetized and operated smoothly. On the first day after the operation, one rabbit died without obvious inducement. In the first week after the operation, two rabbits died due to a lack of food and water. In the $2^{\text {nd }}$ week after the operation, one rabbit demonstrated paralysis of both lower limbs, unable to eat or drink, and died after three days. In the $3^{\text {rd }}$ week after the operation, two rabbits manifested sinus tract, which did not improve after dressing change, decreased activity, and poor appetite in the $4^{\text {th }}$ week, and died one week later. A total of 31 rabbits were selected for modeling.

\section{Characteristics of animal models}

Pathological changes include paravertebral abscess, bone destruction, and sclerotic bone formation at the lesion (Figure 8). After implantation, $\mathrm{CT}$ and $3 \mathrm{D}$ reconstruction can show that the biological substitute is fixed in place at the bone defect (Figure 9).

\section{Drug release of biological substitute in animal models}

INH was released smoothly in the focus vertebrae and paravertebral muscles without sudden release. At the $8^{\text {th }}$ week after implantation, the drug concentration was the lowest and reached the minimum bactericidal concentration (MBC) of INH; the drug concentration in the blood could be maintained for up to one week. The difference in INH concentration in different parts of the same time point was statistically significant (Table 2). The RFP release in the focus vertebral body and paravertebral muscles was sudden release, and it reached the maximum in one week after implantation and the lowest in the focus vertebral body in the $12^{\text {th }}$ week. The RFP concentration in paravertebral muscle was the lowest in the $8^{\text {th }}$ week, and the lowest RFP concentration in focus tissue reached $\mathrm{MBC}$; the RFP concentration in blood could be maintained to $72 \mathrm{~h}$. RFP concentration in different parts of the same time point was statistically significant (Table 3). 

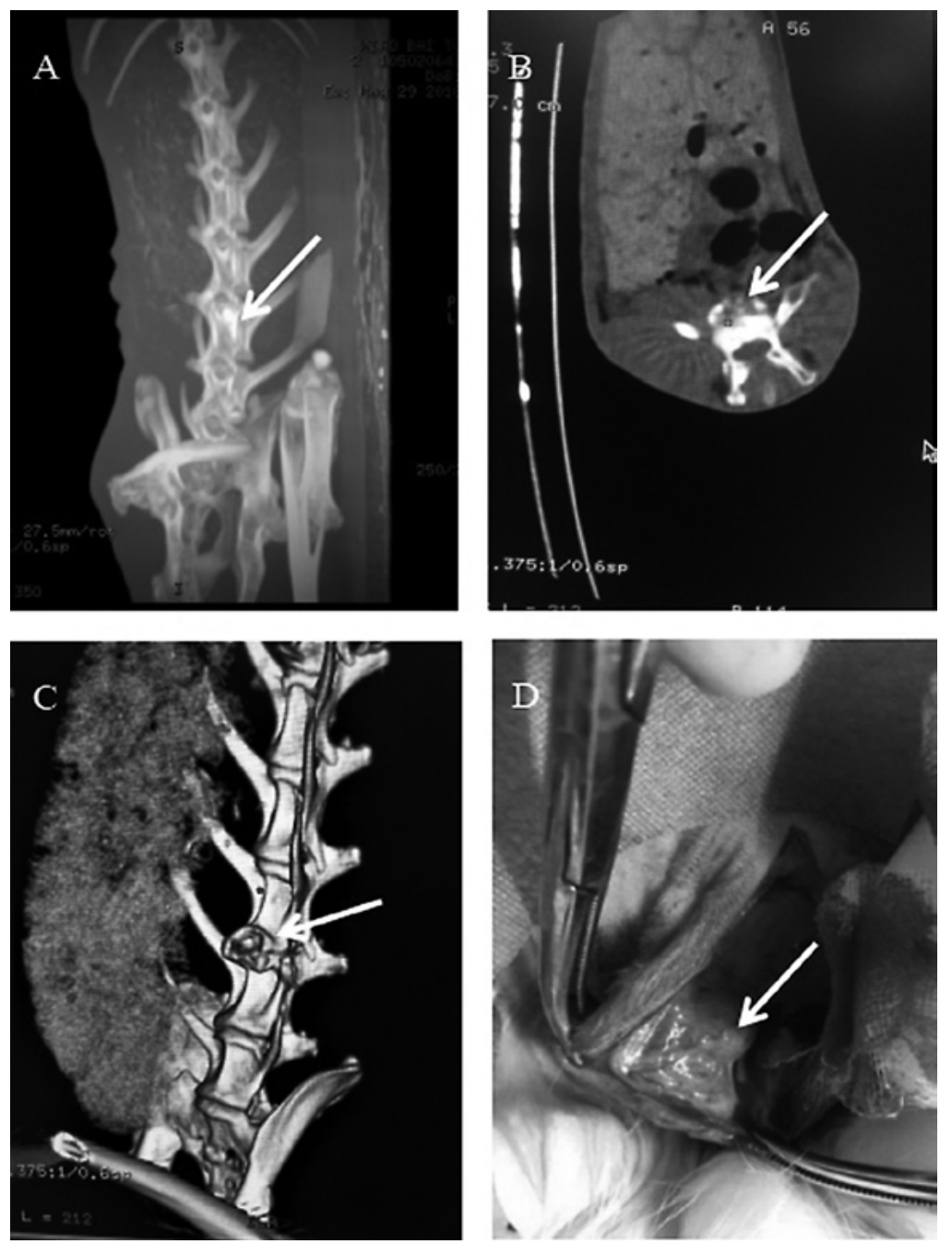

Figure 8. Model of spinal tuberculosis in rabbits. (A-C) CT scan showed a large area of osteolytic destruction, combined with debris sign of L4/5 vertebrae, a sclerotic wall was formed in the osteolytic border (white arrow), (D) The rabbit model showed vertebral body side abscess (white arrow).
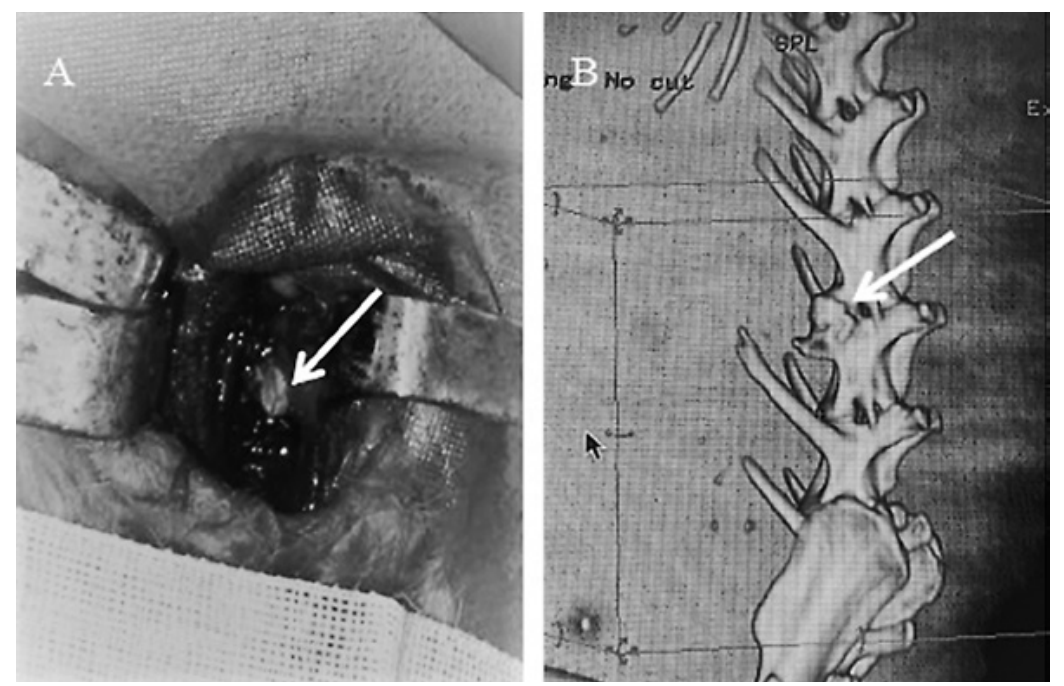

Figure 9. The implantation of a biological substitute. (A, B) The biological substitute was implanted after spinal tuberculosis debridement, $\mathrm{CT}$ and 3D reconstruction showed that the biological substitute was fixed in the defect left by focus (white arrow). 
Table 2. The drug concentration of INH in the biological substitute in an animal model.

\begin{tabular}{|c|c|c|c|}
\hline \multicolumn{5}{|c|}{} & $\begin{array}{c}\text { Focal vertebral body } \\
(\mu \mathrm{g} / \mathrm{mL})\end{array}$ & $\begin{array}{c}\text { Focal paravertebral muscle } \\
(\mu \mathrm{g} / \mathrm{mL})\end{array}$ & $\begin{array}{c}\text { Blood } \\
(\mu \mathrm{g} / \mathrm{mL})\end{array}$ \\
\hline $24 \mathrm{~h}$ & $75.72 \pm 1.35$ & $38.60 \pm 3.30$ & $1.08 \pm 0.24$ \\
\hline $72 \mathrm{~h}$ & $45.77 \pm 2.56$ & $22.72 \pm 1.76$ & $2.25 \pm 0.42$ \\
\hline $1 \mathrm{w}$ & $30.27 \pm 2.26$ & $16.08 \pm 2.52$ & $0.54 \pm 0.22$ \\
\hline $2 \mathrm{w}$ & $22.78 \pm 2.45$ & $10.63 \pm 2.06$ & - \\
\hline $4 \mathrm{w}$ & $8.93 \pm 1.78$ & $3.17 \pm 1.12$ & - \\
\hline $6 \mathrm{w}$ & $5.66 \pm 0.63$ & $1.73 \pm 0.43$ & - \\
\hline $8 \mathrm{w}$ & $2.88 \pm 1.15$ & $0.71 \pm 1.09$ & - \\
\hline $10 \mathrm{w}$ & - & - & - \\
\hline $12 \mathrm{w}$ & - & - & \\
\hline
\end{tabular}

Significant difference $(\mathrm{p}<0.05)$ for INH at the same time in all three tissues.

Table 3. The drug concentration of RFP in the biological substitute in an animal model.

\begin{tabular}{|c|c|c|c|}
\hline \multicolumn{5}{|c|}{ RFP } \\
\hline & $\begin{array}{c}\text { Focal vertebral body } \\
(\mu \mathrm{g} / \mathrm{mL})\end{array}$ & $\begin{array}{c}\text { Focal paravertebral muscle } \\
(\mu \mathrm{g} / \mathrm{mL})\end{array}$ & $\begin{array}{c}\text { Blood } \\
(\mu \mathrm{g} / \mathrm{mL})\end{array}$ \\
\hline $24 \mathrm{~h}$ & $9.66 \pm 1.21$ & $6.73 \pm 1.96$ & $1.06 \pm 0.41$ \\
\hline $72 \mathrm{~h}$ & $20.30 \pm 1.28$ & $21.32 \pm 2.19$ & $0.23 \pm 0.21$ \\
\hline $1 \mathrm{w}$ & $39.40 \pm 2.78$ & $48.70 \pm 1.71$ & - \\
\hline $2 \mathrm{w}$ & $25.66 \pm 1.88$ & $31.49 \pm 2.14$ & - \\
\hline $4 \mathrm{w}$ & $17.47 \pm 2.96$ & $14.69 \pm 2.58$ & - \\
\hline $6 \mathrm{w}$ & $9.27 \pm 2.59$ & $6.83 \pm 1.25$ & - \\
\hline $8 \mathrm{w}$ & $6.60 \pm 2.84$ & $3.73 \pm 1.66$ & - \\
\hline $10 \mathrm{w}$ & $5.67 \pm 1.52$ & - & \\
\hline $12 \mathrm{w}$ & $1.35 \pm 1.20$ & - & \\
\hline
\end{tabular}

Significant difference $(\mathrm{p}<0.05)$ for RFP at the same time in all three tissues.

\section{DISCUSSION}

Due to sequestrum and tissue destruction in the focus of spinal tuberculosis, it is arduous for antituberculosis drugs to reach an effective bactericidal concentration in focus. After sequestrum removal, the vertebral body forms bone defects with different volumes. Due to the hardness and diffuseness of sclerotic vertebral bone, its repair is challenging (1). Based on the previous research, this study further explores the release characteristics of biological substitutes in vitro and in animal models (6).

At present, the frequently used biological substitutes for bone tissue include hydroxyapatite, calcium phosphate, and calcium sulfate (8-9). The chemical composition and structure of these compounds are similar to that of human bone, which is conducive to bone regeneration (10-11). There have been studies to prepare a sustained-release carrier of antituberculosis by loading calcium phosphate with RFP.
Although sustained-release time can be maintained to 12 weeks, there are shortcomings, such as single drug loading, easy to produce drug resistance, and weak compressive strength (12). Anti-infective drugs are used in combination with low pore calcium phosphate, which obtained better support performance after being implanted into rabbit tibia, but the drug release rate became difficult to control with the increase of time (13). Like calcium phosphate, nHA exhibits high solubility and biological activity and can better integrate with bone, but it has a brittle texture and weak compressive strength (14-15). However, nHA and CS compounds can overcome some of their shortcomings and form new biomaterials in a particular proportion. The nHA-CS composites have a weak rejection response to living organisms, promote bone growth under the periosteum, and adsorb biological factors and drugs (16).

Herein, nHA-CS composite material with supporting properties was employed to load 
antituberculosis drugs for experiments. If calcium and phosphorus materials were used alone in the system of bone tissue repair materials, the slow-release effect would be reduced, and body fluid $\mathrm{pH}$ would be increased (17). Therefore, natural polymer materials such as gelatin, alginate, CTS, and other materials should be added for modification. The high swelling property of gelatin can affect the mechanical strength of hydroxyapatite gelatin composite (18). To increase the mechanical strength of a composite, cross-linking is required. However, most of the cross-linking agents have various biological toxicity degrees, and the manufacturing process is complex. These factors limit the combination and application of gelatin and hydroxyapatite. CTS is a natural hydrophilic polysaccharide, non-toxic and degradable, with good biocompatibility. Because of its own viscosity, CTS can increase the stability and slow-release performance of bone repair materials (19). CTS can increase the water absorption of the HA complex and the exchange efficiency of humoral environment substances (20). A control study between the groups found that composite material added with CTS can maintain the drug concentration in the rabbit osteomyelitis model for 45 days (21).

When a biological substitute releases the drug into the body, the drug is continuously diffused and consumed in a dynamic cycle due to body fluids' infiltration, so it is essential to select an appropriate method to evaluate in vitro release characteristics of the biological substitute. In this study, we used the complete exchange method that has been reported (22). The purpose is to simulate the release environment of biological substitutes in the body, and measured drug concentration at each time point is not affected by the previous time point. As a watersoluble antituberculosis drug, INH exhibits a strong bactericidal ability against metabolically active mycobacterium tuberculosis in focus, and its minimum inhibitory concentration (MIC) in vitro is 0.005 to $0.5 \mu \mathrm{g} / \mathrm{mL}$. As a fat-soluble antituberculosis drug, RFP belongs to broad-spectrum antibiotics, with MIC of $0.025-0.05 \mu \mathrm{g} / \mathrm{mL}$. MBC of the two drugs is 10 times MIC (23). According to the results of in vitro drug release, INH has an obvious burst release. The reason may be that INH, as a hydrophilic drug, will seep into the superficial part of biological substitute with water, causing uneven drug distribution. Besides, the sustained-release effect of CTS on the fat-soluble drug RFP is stronger than that of watersoluble drug INH. However, RFP showed a relatively stable release, without obvious burst release.

In the model rabbits, INH's drug concentration was maintained in focus vertebra and paravertebral muscles until week 8 , and both reached MBC. In the first week, RFP concentration in focus vertebra and paravertebral muscles reached the highest level, and MBC was still reached in the $12^{\text {th }}$ week. The sudden release of INH four weeks ago was relatively obvious. Considering that CTS is a hydrophilic substance and INH is also a water-soluble drug, its slow-release effect on INH is slightly weaker. On the other hand, for an infectious disease of spinal tuberculosis, large and rapid drug release in the early stage plays an important role in controlling infection and improving prognosis (24). The RFP release in biological substitute increased gradually before one week, which may be because RFP is a fat-soluble drug, which is opposite to CTS solubility, so CTS has a strong slow-release effect on the early stage of RFP. However, after one week, RFP showed stable and sustained drug release characteristics, considering that composite material gradually became loose and degraded with body fluid infiltration and decreased CTS concentration. INH and RFP concentrations in venous blood were maintained for one week and three weeks, respectively, and less than that in the focus vertebra and muscle tissue. The reasons may be as follows: due to the slow-release effect of biological substitute, a fewer amount of drugs enter local circulation by osmosis, but it is difficult to accumulate after metabolism of liver and kidney, and inflammatory reaction zone, as well as scar tissue formed after biological substitute implantation, will also hinder the drug from entering the blood circulation. The drugs in biological substitute directly contact and spread in the focus area, playing the role of consumption and anti-infection, reducing drugs that can enter the blood. Such release characteristics are conducive to reduce side effects caused by systemic chemotherapy. In general, INH in the material appears as a rapid release phase and a slow-release phase in the early and late stages, respectively. This dual-phase release feature can maximize its early sterilization advantages. The drug concentration of RFP before one week showed a gradual increase, and it was released smoothly in the later period. Besides, RFP has strong antibacterial activity against tubercle bacteria that grow slowly and intermittently. Therefore, this release curve can better apply RFP in antituberculosis treatment.

The shortcomings of this study: due to the tiny volume of rabbits' vertebral body, it is difficult to obtain a sufficient amount of various tissues of focal vertebrae, such as sclerotic bones and subnormal bones, when taking samples. Only the release characteristics of biological substitutes in vitro and in vivo have been verified, and the therapeutic effects of other aspects have not been further confirmed. 


\section{CONCLUSION}

In summary, biological substitutes loaded with antituberculosis drugs can achieve good sustainedrelease effects in vitro, effectively release drugs locally in rabbit spinal tuberculosis animal models, and reach effective drug concentrations in lesions.

\section{ACKNOWLEDGMENTS}

This study is supported by the National Natural Science Foundation of China (No. 8196090244) and Ningxia Natural Science Foundation (No. 2018AAC03138).

\section{CONFLICT OF INTEREST}

The authors report no conflicts of interest.

\section{REFERENCES}

1. Ge Z.H., Wang Z.L., Wei M.J.: Eur. Spine J. 17, 1482 (2008).

2. Liu P., Jiang H., Li S.J., Lin Z., Jiang J.M.: J. Orthop. Res. 35, 200 (2016).

3. Huang D., Li D.W., Wang T.T., Shen H., Zhao P., et al.: Biomaterials. 52, 417 (2015).

4. Lin L.X., Hao R.S., Xiong W., Zhong J.: J. Biosci. Bioeng. 119, 591 (2015).

5. Saranya N., Saravanan S., Moorthi A., Ramyakrishna B., Selvamurugan N.: J. Biomed. Nanotechnol. 7, 238 (2011).

6. Ma H., Cai Z.C., Liang S.M., Ma R., Yin F., et al.: Chin. J. Spine Spinal Cord. 28, 641 (2018) (in Chinese).

7. Chen Z., Wu P., Ma R., Xu G.X., Zhang H.Y., et al.: J. Ningxia Med. Univ. 37, 216 (2015) (in Chinese).
8. Fan X.X., Peng H.T., Li H., Yan Y.G.: J. Biomater. Sci. Polym. Ed. 30, 107 (2019).

9. Dai Z.Y., Li Y., Lu W.Z., Jiang D.M., Li Y., et al.: Int. J. Nanomedicine. 10, 6303 (2015).

10. Liu Y., Luo D., Wang T.: Small. 12, 4611 (2016).

11. Qiu G.T., Shi Z.J., Zhao L.: Orthop. J. China. 24, 1406 (2013) (in Chinese).

12. Ye X.Y., Zhen P., Li X.F., Zhao D.H., Gao M.X.: Orthop. J. China. 8, 1981 (2010) (in Chinese).

13. Kundu B., Soundrapandian C., Nandi S.K., Mukherjee P., Dandapat N., et al.: Pharm. Res. 27, 1659 (2010).

14. Chaudhry A.A., Yan H.X., Gong K.N., Inam F., Viola G., et al.: Acta Biomater. 7, 791 (2011).

15. Liao J.G., Li Y.Q., Duan X.Z., Zhu L.L.: Prog. Chem. 27, 220 (2015) (in Chinese).

16. Ge L., Gou S.H., Yang S.C., Wang S.L.: Acad. J. Second Military Med. Univ. 27, 1121 (2006) (in Chinese).

17. Loi F., Córdova L.A., Pajarinen J., Lin T.H., Yao Z.Y., et al.: Bone. 86, 119 (2016).

18. Foox M., Zilberman M.: Expert Opin. Drug Deliv. 12, 1547 (2015).

19. Sun Y., Li X.Y., Liang X.F., Wan Z.Y., Duan Y.R.: J. Nanosci. Nanotechnol. 13, 5260 (2013).

20. Liang W.H., Tan Z.J., Qu J.S., Zhao H.X.: J. Funct. Mater. 46, 134 (2015) (in Chinese).

21. Shi P.J., Zuo Y., Li X.W., Zou Q., Liu H.H., et al.: J. Biomed. Mater. Res. A. 93, 1020 (2010).

22. McLaren A.C., McLaren S.G., Nelson C.L., Wassell D.L., Olsen K.M.: Clin. Orthop. Relat. Res. 403, 54 (2002).

23. Jutte P.C., Rutgers S.R., Van Altena R., Uges D.R., Van Horn J.R.: Int. J. Tuberc. Lung Dis. 8, 1368 (2004).

24. Fan S.W., Hu Z.A.: China J. Orthop. Trauma. 30, 783 (2017) (in Chinese). 